SFB

Model based optimization of a statistical simulation model

823

for single diamond grinding

Swetlana Herbrandt, Uwe Ligges,

Manuel Pinho Ferreira, Michael Kansteiner,

Dirk Biermann, Wolfgang Tillmann, Claus Weihs

Nr. 11/2016

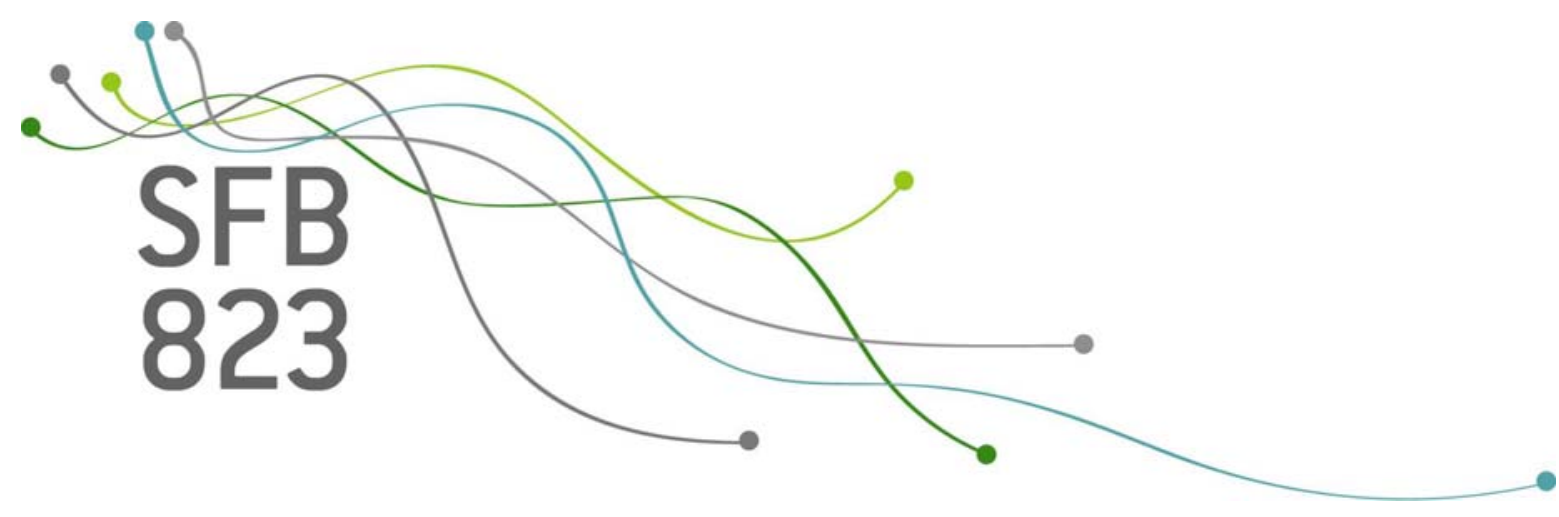





\title{
Model Based Optimization of a Statistical Simulation Model for Single Diamond Grinding
}

\author{
Swetlana Herbrandt • Uwe Ligges · Manuel \\ Pinho Ferreira • Michael Kansteiner • Dirk \\ Biermann • Wolfgang Tillmann · Claus \\ Weihs
}

Received: date / Accepted: date

\begin{abstract}
We present a model for simulating normal forces arising during a grinding process in cement for single diamond grinding. Assuming the diamond to have the shape of a pyramid, a very fast calculation of force and removed volume can be achieved. The basic approach is the simulation of the scratch track. Its triangle profile is determined by the shape of the diamond. The approximation of the scratch track is realized by stringing together polyhedra. Their sizes depend on both the actual cutting depth and an error implicitly describing the material brittleness. Each scratch track part can be subdivided into three three-dimensional simplices for a straightforward calculation of the removed volume. Since the scratched mineral subsoil is generally inhomogeneous, the forces at different positions of the workpiece are expected to vary. This heterogeneous nature is considered by sampling from a Gaussian random field.

To achieve a realistic outcome the model parameters are adjusted applying model based optimization methods. A noisy Kriging model is chosen as surrogate to approximate the deviation between modelled and observed forces. This deviation is minimized and the results of the modelled forces and the actual forces from conducted experiments are rather similar.
\end{abstract}

Keywords noisy Kriging · augmented expected improvement · MBO

S. Herbrandt, U. Ligges, C. Weihs

TU Dortmund

Department of Statistics

E-mail: [herbrandt, ligges, weihs]@statistik.tu-dortmund.de

M. P. Ferreira, W. Tillmann

TU Dortmund

Institute of Materials Engineering

E-mail: manuel.ferreira@tu-dortmund.de

E-mail: wolfgang.tillmann@tu-dortmund.de

M. Kansteiner, D. Biermann

TU Dortmund

Institute of Machining Technology

E-mail: michael.kansteiner@tu-dortmund.de

E-mail: biermann@isf.de 


\section{Introduction}

Core drilling with diamond impregnated tools is a widely used method in the construction industry. The various components of the machined material and the tool results in a very complex process. For better understanding of this process simulation models are developed in addition to conducted experiments. Due to the high complexity, the models and experiments often focus on a part of the process to reduce the number of influencing factors. Brinksmeier et al. [3] give an overview about the various models for the description of forces, material removal and temperature in grinding processes. Despite the existence of various models, the many different constellations of material, tool production parameters and the kinds of processes result in a need for further research.

In this work we will focus on the forces arising in a grinding process with a pyramidal diamond. Inspired by the work of Raabe et al. ([13], [14], [15]) we derive a force model based on the removed material volume and the material heterogeneity (see sec. 3). Contrary to the model of Raabe et al., we reduce the simulation of the complete workpiece to the scratch track produced by the diamond in order to reduce the time complexity (see discussion in sec. 4).

To achieve realistic outcome the model parameters are adjusted in order to minimize the deviation between the modelled and observed forces of conducted experiments (see sec. 2). For the parameter adjustment, model based optimization methods for stochastic black box functions are applied, in which the chosen deviation measure is approximated by the noisy Kriging model (sec. 5). Since the results of the optimization are very promising, the optimized model is used for the optimization of the drilling process (sec. 6). Thereby, the two process parameters cutting speed and feed speed are optimized in order to minimize the work needed to drill a predefined total depth.

\section{Experiments}

In core drilling a diamond tipped drill core bit consisting of several segments is used. These rectangular segments are manufactured in a powder metallurgical process route by sintering a mixture of diamonds and metal powder. The finished segments are attached to a circular body in equally spaced intervals. During the drilling process the role of the metal matrix surrounding the diamonds is to protect the diamonds against an early break out, whereas the diamonds remove the material.

Since in the core drilling process many different factors influence the strength of the arising forces [4], experiments with only one diamond are conducted. The so called single grain scratch tests keep the number of influencing factors small due to the absence of diamond break outs, interactions between diamonds and the influence of the metal matrix surrounding the diamonds in a segment. A further reduction of influencing factors is carried out by the application of dressing diamonds, which have the shape of a pyramid with angle $\alpha=110^{\circ}$ (fig. 1(a)). During the test a dressing diamond scratches into the material sample on a circular path with radius $r[\mathrm{~mm}]$, a constant feed speed $v_{f}\left[\frac{\mathrm{mm}}{\mathrm{min}}\right]$ and a constant cutting speed $v_{c}\left[\frac{\mathrm{m}}{\mathrm{min}}\right]$ (fig. $1(\mathrm{~b})$ ). We consider the 16 speed combinations of the $4^{2}$-full factorial design with the factor levels $v_{c} \in\{40.5,117,193.5,270\}$ and $v_{f} \in\{2,4.5,7,9.5\}$. 
On each of five circular disks (material samples) twelve scratch tests can be conducted using the radii $r \in\{16,17, \ldots, 27\}$. Since scratch tests are destructive tests repetitions on the same sample with the same radius and speed combination are not possible. Therefore, we use every second radius for the repetition of the speed combination applied on the adjacent radius. That means that the two experiments on the radii $r$ and $r+1$ for $r \in\{16,18,20,22,24,26\}$ have the same speed parameters $\left(v_{c}, v_{f}\right)$. We allocate the 16 speed combinations to the six blocks (number of radii without radii for repetitions) of size five (number of material samples) by optimizing the $D$-criterion [16]. Let denote $\mathcal{R}\left(v_{c}, v_{f}\right)$ the set of radii with the same speed combination $\left(v_{c}, v_{f}\right)$ and $n_{\mathcal{R}}\left(v_{c}, v_{f}\right)$ the number of elements in this set. Since each speed combination is repeated on the adjacent radius, each set contains at least two elements.

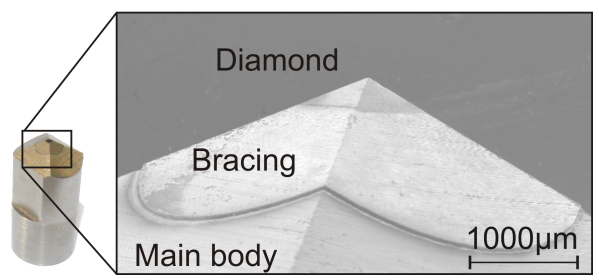

(a)

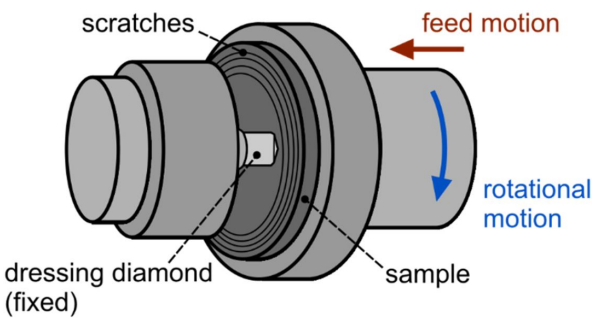

(b)

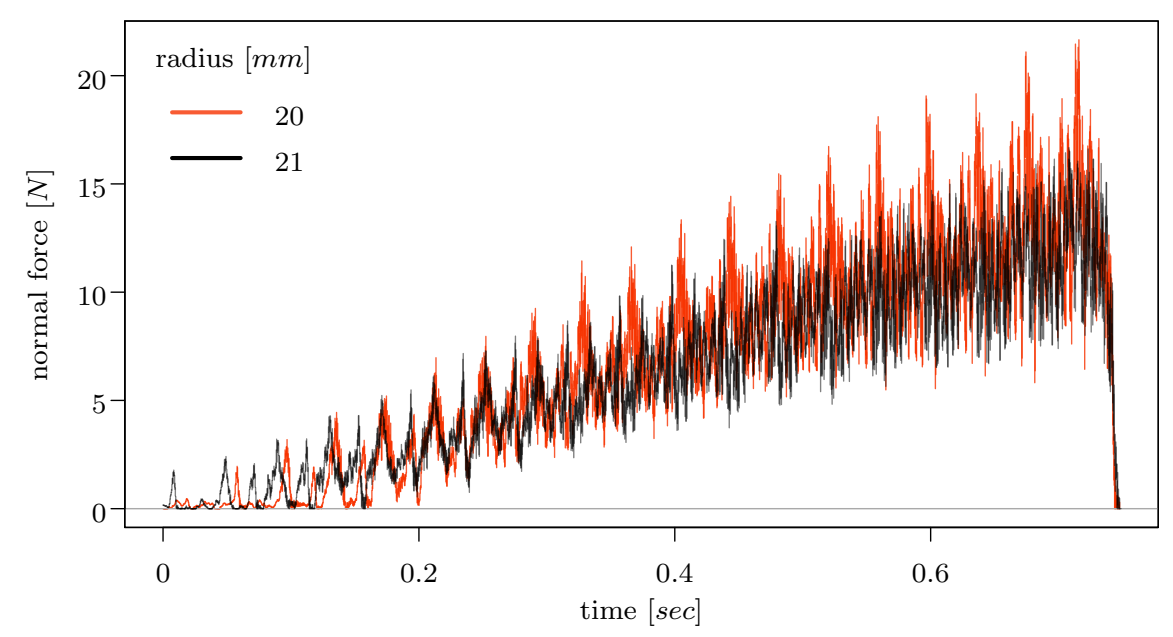

(c)

Fig. 1 (a) Dressing diamond, (b) experimental setup for single diamond scratching, (c) normal forces on cement for the speed combination $v_{c}=193.5 \frac{\mathrm{m}}{\mathrm{min}}, v_{f}=7 \frac{\mathrm{mm}}{\mathrm{min}}$.

During each experiment the arising normal forces are recorded with a sampling rate of $200 \mathrm{kHz}$. The experiment is terminated when a total depth of $0.08 \mathrm{~mm}$ is reached. Since the diamond is pyramidal, the profile of the resulting scratch track has the shape of a triangle. If the diamond only removes the material in front of it, the removal mechanism is exact and the resulting scratch track profile is a 
triangle with a height of $h=0.08 \mathrm{~mm}$ and a base width of $w=2 h \tan \frac{\alpha}{2}$. This removal mechanism can be observed on ductile materials, e.g. steel. Mineral substrates, like basalt or cement, tend to brittle fracture and therefore generally show a more irregular removal. In the experiments, we consider the materials basalt, cement, concrete (mixture of cement and basalt), steel and reinforced concrete. The described design of experiments is applied to each material.

The resulting normal forces show a linear increase in time and an increase in variance (see fig. 1(c)). Both characteristics can be explained with the shape of the used diamond and the increasing contact area between workpiece and diamond during the experiment. The seasonal component has two causes. Material inhomogeneity leads to different force values. Since the diamond scratches on a circular path this pattern is repeated in each revolution in more or less the same way depending on the degree of the occurring inhomogeneity. Moreover, the sinusoidal course can be explained by the workpiece tilting. This phenomenon is particularly well detectable at the beginning of the force time series when the signal temporarily drops to zero, because the contact between workpiece and diamond is interrupted.

\section{Simulation Model}

In this section we will introduce a model for the simulation of normal forces $F_{t}, t \in[0, T]$, arising during a scratch test with the known parameter settings $\left(v_{c}, v_{f}, r\right)$. Assuming that the majority of the time series course can be explained with the material heterogeneity and the contact area between diamond and workpiece (sec. 2) or removed volume, respectively, we model the normal forces

$$
\mathcal{F}_{t}=\frac{g_{z v}}{r} \cdot z_{t} \cdot v_{t}+\frac{g_{v}}{r} \cdot v_{t}, \quad t \in[0, T]
$$

as a sum of removed volume $v_{t}$ and the interaction $z_{t} v_{t}$ of removed volume and material heterogeneity $z_{t}$. In the following implementation we consider the discretization in time $t_{i}(i=0, \ldots, N \nu)$ with $t_{0}=0$ and $t_{N \nu}=T$, where $N$ is the number of revolutions and $\nu$ the number of simulated observations per revolution.

The basic approach is the approximation of the scratch track produced by the dressing diamond. For this purpose we place $\nu$ equally spaced triangles on the diamond's circular path with radius $r$ for each revolution (fig. 2(a)). By connecting each two adjacent triangles we can form a scratch track part representing one of our observations (see fig. 2(b)). To complete the last revolution we have to place a further triangle at the identical angle on the circular path as the first triangle, after which $N \nu+1$ scratch track triangles are placed in total for $N$ revolutions with $\nu$ observation. Let $\mathcal{D}=\left\{D_{j} \mid 1 \leq j \leq \nu N+1\right\}$ denote the set of all these triangles. The size of the $j$ th $(j=1, \ldots, N \nu+1)$ triangle

$$
D_{j}=\left(\begin{array}{c}
d_{3 j-2} \\
d_{3 j-1} \\
d_{3 j}
\end{array}\right)=\left(\begin{array}{ccc}
\left(-w_{j}+r\right) \cdot \cos \left(k_{j}\right) & \left(-w_{j}+r\right) \cdot \sin \left(k_{j}\right) & 0 \\
\left(w_{j}+r\right) \cdot \cos \left(k_{j}\right) & \left(w_{j}+r\right) \cdot \sin \left(k_{j}\right) & 0 \\
r \cdot \cos \left(k_{j}\right) & r \cdot \sin \left(k_{j}\right) & -h_{j}
\end{array}\right) \in \mathbb{R}^{3 \times 3}
$$

with the half width $w_{j}=\tan \left(\frac{\alpha}{2}\right) h_{j}$ at the angular frequency $k_{j}=\frac{2 \pi}{\nu}[(j-1) \bmod \nu]$ basically depends on the intrusion depth $h_{j}$ and the angle $\alpha$ of the diamond. The increase of the intrusion depth per observation can be calculated as 
$a=\frac{v_{f}}{v_{c} \nu} 2 \pi r 10^{-3}[\mathrm{~mm}]$. If the removal mechanism is realized exactly as described in section 2 , the $j$ th intrusion depth is just $h_{j}=a(j-1)$. To enable the simulation of brittle material the intrusion depth is modelled as $h_{j}=a(j-2)+a_{j}^{\star}$ for $j=2, \ldots, \nu N+1\left(h_{1}=0\right)$, where $a_{2}^{\star}, \ldots, a_{N \nu+1}^{\star}$ are i.i.d. Beta $(0, a \nu, p, q)$ distributed intrusion depth innovations. The parameters of this generalized beta distribution determinate the degree of the material brittleness. While the first two parameters limit the intrusion depths to the interval $[0, a \nu]$, the parameters $p$ and $q$ specify the shape of the distribution.

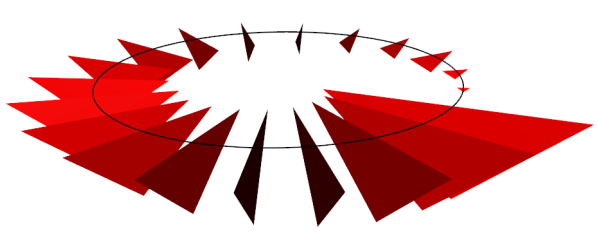

(a)

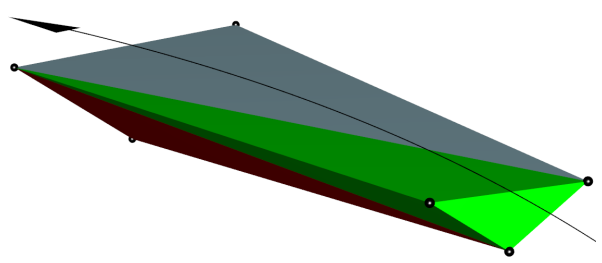

(b)

Fig. 2 (a) Scratch track triangles for the first revolution and (b) tessellation of one scratch track part into three simplices (red, green and blue).

For the approximation of the removed volume, the space between each two adjacent triangles and thus the volume of each scratch track part is considered. The volume of the $i$ th scratch track part can be determined by subdividing the space between the corresponding triangles $D_{i}$ and $D_{i+1}$ into three 3 -dimensional simplices (fig. 2(b)). Let

$$
S_{i}=\left(\begin{array}{c}
s_{3 i-2} \\
s_{3 i-1} \\
s_{3 i}
\end{array}\right)=\left(\begin{array}{cccc}
3 \cdot i-2 & 3 \cdot i-1 & 3 \cdot i & 3 \cdot i+1 \\
3 \cdot i+1 & 3 \cdot i+2 & 3 \cdot i+3 & 3 \cdot i-1 \\
3 \cdot i & 3 \cdot i-1 & 3 \cdot i+3 & 3 \cdot i+1
\end{array}\right) \in \mathbb{N}^{3 \times 4}
$$

be the matrix with the indices of the triangle points corresponding to the simplices. Since the volume of a 3 -dimensional simplex with vertex indices $s_{l}=$ $\left(s_{l 1}, s_{l 2}, s_{l 3}, s_{l 4}\right), l=1, \ldots, 3 N \nu$, can be calculated as

$$
V\left(s_{l}\right)=\frac{1}{3 !}\left|\operatorname{det} M_{l}\right| \text { with } M_{l}=\left(\begin{array}{ll}
1 & d_{s_{l 1}} \\
1 & d_{s_{l 2}} \\
1 & d_{s_{l 3}} \\
1 & d_{s_{l 4}}
\end{array}\right)=\left(\begin{array}{llll}
1 & d_{s_{l 1}, 1} & d_{s_{l 1}, 2} & d_{s_{l 1}, 3} \\
1 & d_{s_{l 2}, 1} & d_{s_{l 2}, 2} & d_{s_{l 2}, 3} \\
1 & d_{s_{l 3}, 1} & d_{s_{l 3}, 2} & d_{s_{l 3}, 3} \\
1 & d_{s_{l 4}, 1} & d_{s_{l 4}, 2} & d_{s_{l 4}, 3}
\end{array}\right)
$$

we can derive the volume of this scratch track part as

$$
\begin{aligned}
V\left(S_{i}\right)=V( & \left(s_{3 i-2}\right)+V\left(s_{3 i-1}\right)+V\left(s_{3 i}\right) \\
=\frac{1}{3 !}[ & \left|2 h_{i} w_{i}\left(w_{i+1}-r\right)\right|+\left|2 h_{i+1} w_{i+1}\left(w_{i}+r\right)\right| \\
& \left.\quad+\left|h_{i+1} w_{i}\left(w_{i+1}-r\right)-h_{i} w_{i+1}\left(w_{i}+r\right)\right|\right] \sin \left(\frac{2 \pi}{\nu}\right)
\end{aligned}
$$


with $w_{i}=\tan \left(\frac{\alpha}{2}\right) h_{i}$. After the first revolution the previously removed volume has to be considered, so that the effectively removed volume is obtained by

$$
v_{i}=\left\{\begin{array}{ll}
0, & i=0 \\
V\left(S_{i}\right), & i \leq \nu \\
V\left(S_{i}\right)-V\left(S_{i-\nu}\right), & i>\nu
\end{array} \quad(i=0, \ldots, N \nu) .\right.
$$

Since the scratch track is a model of the removed material, each point of the scratch track can be considered as a material sample at the corresponding point of the workpiece. Therefore, the material heterogeneity can be taken into account by sampling values for each point from the process

$$
Z(d)=\mu+G(d)+\xi,
$$

where $G$ is a Gaussian process with $E(G(d))=0, \operatorname{Var}(G(d))=\sigma^{2}$, exponential correlation function

$$
R_{i j}=\operatorname{Cor}\left(G\left(d_{i}\right), G\left(d_{j}\right)\right)=\exp \left(-\frac{\left\|d_{i}-d_{j}\right\|}{\psi}\right)
$$

and $\xi \sim N\left(0, \sigma_{\xi}^{2}\right)$. The range parameter $\psi$ states the strength of the correlation of two variables regarding their distance from each other. Thus, lower values of $\psi$ result in smaller correlations which in turn can be interpreted as a higher degree of heterogeneity. Conversely, a big range allows the modelling of uniform material structure without sudden changes. The range parameter is only relevant, if the variance $\sigma^{2}$ is large enough. For the heterogeneity model of the $i$ th observation the mean value

$$
z_{i}=\left\{\begin{array}{ll}
0, & i=0 \\
\frac{1}{6} \sum_{j=3 \cdot i-2}^{3 \cdot i+3} Z\left(d_{j}\right), & i>0
\end{array} \quad(i=0, \ldots, N \nu)\right.
$$

is computed (fig. 3(a)). Figure 3(b) shows a scratch track of the first revolution with $\nu=100$ observations and heterogeneity values $z_{1}, \ldots, z_{100}$ represented by different color shades.

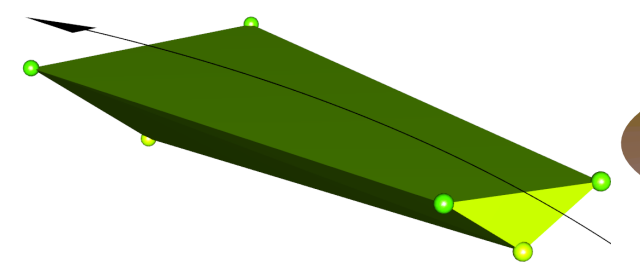

(a)

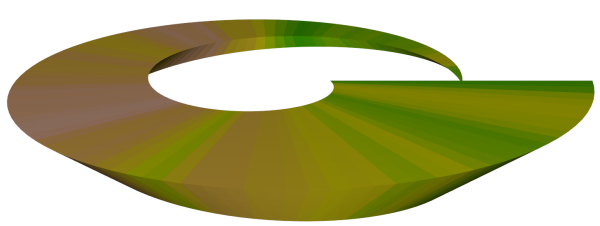

(b)

Fig. 3 (a) Scratch track part with sampled heterogeneity values for the six points represented by different color shades for the points and the mean value (color of track part) and (b) scratch track of the first revolution with $\nu=100$ observations (scratch track parts) with assigned heterogeneity values.

In our force model the removed volume $v_{t}$ determines the slope of the resulting forces. Since the volume of a scratch track part depends on the resolution $\nu$ of the 
simulation, the volumes are weighted by the factor $g_{v}$, which has to be estimated. For the same reasons, the interaction $z_{i} v_{i}$ is multiplied with the factor $g_{z v}$. By the multiplication of the heterogeneity time series with the volume time series, we achieve a continuous increase in the variance of the modelled force.

\section{Comparison of time complexity}

In this section we discuss the time complexity of the presented scratch track model, the Raabe et al. ([13], [14], [15]) model and the Herbrandt et al. [5] model, which includes first simplifications of the Raabe et al. [13] model in order to reduce the time complexity.

In the model of Raabe et al. ([13], [14], [15]) the workpiece is simulated with two sets of $n_{R}$ and $k_{R}$ points, where $n_{R}<k_{R}$ (coarse and fine lattice). For each point of the first set a value from a Gaussian random field (time complexity $O\left(n_{R}^{3}\right)$, [9]) is sampled. The values for the points of the finer lattice are interpolated from the coarser lattice with ordinary Kriging (time complexity $O\left(n_{R}^{3}+n_{R} k_{R}\right)$, [9]). The workpiece (as well as the diamond) is described by triangulating the corresponding point sets of size $k_{R}\left(m_{R}\right.$ for the diamond) into 3-dimensional simplices by applying the Delaunay tesselation $\left(O\left(k_{R}^{2}\right)\right.$ and $O\left(m_{R}^{2}\right)$, respectively, [10]). The interaction between diamond and workpiece is determined by examining if one of the workpiece points is inside of one of the diamond simplices $\left(O\left(I_{R} k_{R} m_{R}^{2}\right)\right)$. This results in a worst case time complexity of $O\left(n_{R}^{3}+n_{R} k_{R}+k_{R}^{2}+I_{R} k_{R} m_{R}^{2}\right)$. To reduce this complexity we developed a model where only the workpiece surface is modelled with $k_{H}$ points [5]. This approach reduced the worst case complexity to $O\left(n_{H}^{3}+n_{H} k_{H}+I_{H} k_{H} m_{H}^{2}\right)$, since we do not tesselate the $k_{H}$ points of the finer lattice. Additionally, the typically used numbers $n_{H}, m_{H}$ and $I_{H}$ are smaller than the numbers in the Raabe et al. model (see table 1).

Table 1 Typically used numbers of the modelled observations $I$, points in coarse lattice $n$, points in fine lattice $k$ and points in diamond $m$ for the three models.

\begin{tabular}{lllll}
\hline & & Raabe et al. [13] & Herbrandt et al. [5] & Scratch track based model \\
\hline process & $I$ & $I_{R}=57803$ & $I_{H}=6800$ & $I_{S}=3400$ \\
\multirow{2}{*}{ workpiece } & $n$ & $n_{R}=6500$ & $n_{H}=496$ & $n_{S}=10203$ \\
\multirow{2}{*}{ tool } & $k$ & $k_{R}=9400$ & $k_{H}=16960$ & - \\
\hline
\end{tabular}

The scratch track based model presented in this section needs the sampling from the Gaussian random field for the $n_{S}=3 I_{S}+3$ points, where $I_{S}=\nu N$, and thus has a time complexity of $O\left(n_{S}^{3}+I_{S}\right)$. From the numbers of table 1 it seems that the scratch track based model has the worst runtime. In the practical application, however, the cubic term of the random field generation does not dominate the execution time. For the two faster models table 2 shows the average total runtimes for the computation of the models and the average runtimes for the generation of the Gaussian random field. In the scratch track based model about $85 \%$ of the time is used for the random field. Despite the high number of points $n_{S}$, the computation is done in less than two seconds. In the Herbrandt et al. [5] 
model the random field part accounts for less than $1 \%$ of the total runtime. This small percentage is firstly due to the small number $n_{H}$, but especially it results from the high computation time in total.

Table 2 Average runtimes (in seconds) for the generation of the Gaussian random field and the computation of total model.

\begin{tabular}{lll}
\hline & Herbrandt et al. [5] & Scratch track based model \\
\hline Gaussian random field & 0.055 & 1.58 \\
total & 367 & 1.93 \\
\hline
\end{tabular}

The difference between the total runtimes of the two models suggests that the generation of the Gaussian random field is not the dominant factor concerning the runtime of the Herbrandt et al. model. Therefore, applying the presented method is faster by orders of magnitude.

\section{Model based Optimization}

To obtain a realistic outcome, the model parameters $\theta=\left(g_{z}, g_{v}, \mu, \sigma^{2}, \sigma_{\xi}^{2}, \psi, p, q\right)^{T} \in$ $\Theta \subset \mathbb{R}^{8}$ have to be adjusted. The forces from the conducted experiments (see sec. 2) are used as reference. To achieve an appropriate correspondence between modelled and observed forces, the parameters are adjusted for each speed combination. For this purpose the expected deviation

$$
E\left(\left\|f\left(v_{c}, v_{f}, r\right)-\mathcal{F}(\theta, r)\right\|_{D}\right)=E\left(D\left(f\left(v_{c}, v_{f}, r\right), \mathcal{F}(\theta, r)\right)\right)
$$

of a measured force $f\left(v_{c}, v_{f}, r\right)$ from the model force $\mathcal{F}(\theta, r)$ is minimized. By estimating the expectation with the arithmetic average of $M$ (here: $M=25$ ) realizations $F$ of the force model $\mathcal{F}$ and $n_{\mathcal{R}}\left(v_{c}, v_{f}\right)$ observed forces $f\left(v_{c}, v_{f}, r\right)$ with radii $r \in \mathcal{R}\left(v_{c}, v_{f}\right)$, the optimal parameter settings for one speed combination are obtained as

$$
\begin{aligned}
& \theta^{\star}\left(v_{c}, v_{f}\right)=\underset{\theta \in \Theta}{\arg \min } \bar{D}\left(f\left(v_{c}, v_{f}\right), F(\theta)\right) \\
& \quad=\underset{\theta \in \Theta}{\arg \min } \frac{1}{3 M n_{\mathcal{R}}\left(v_{c}, v_{f}\right)} \sum_{r \in \mathcal{R}\left(v_{c}, v_{f}\right)} \sum_{m=1}^{M}\left[d_{R}\left(\widetilde{f}\left(v_{c}, v_{f}, r\right), \widetilde{F}(\theta, r)\right)\right. \\
& \left.\quad+d_{\beta}\left(f^{\star}\left(v_{c}, v_{f}, r\right), F^{\star}(\theta, r)\right)+d_{S}\left(\tilde{f}\left(v_{c}, v_{f}, r\right), \widetilde{F}(\theta, r)\right)\right],
\end{aligned}
$$

where the terms are discussed in the following. The considered deviation measure $\bar{D}$ is the mean of measures for the comparison of the three characteristics slope, range, and spectrum. For the comparison the forces $f=f\left(v_{c}, v_{f}, r\right)=$ $\left\{f_{t_{i}}\left(v_{c}, v_{f}, r\right) \mid 0 \leq t_{i} \leq T_{f}, i=1, \ldots, L, L\right.$ number of observations $\}$ and $F=$ $F(\theta, r)=\left\{F_{t_{i}}(\theta, r) \mid 0 \leq t_{i} \leq T_{F}, i=1, \ldots, N \nu\right\}$ have to be aligned. Due to the different sampling rates and since the sampling rate of $f$ is very high, we decide to exploit the characteristics of the time series, rather than applying very time 
consuming methods like the dynamic time warping. Therefore, the forces $f$ and $F$ are aligned by the intercepts of the corresponding linear models

$$
f=\beta_{f 0}+\beta_{f 1} t+\varepsilon_{f} \text { and } F=\beta_{F 0}+\beta_{F 1} t+\varepsilon_{F} .
$$

Therefore, the force with the smaller estimated intercept $\left(\widehat{\beta}_{f 0}\right.$ or $\left.\widehat{\beta}_{F 0}\right)$ is shifted by redefining the starting time

$$
f^{\star}= \begin{cases}f, & \widehat{\beta}_{f 0}>\widehat{\beta}_{F 0} \\ \left\{f_{t_{i}} \mid 0 \leq t_{i}-\frac{\widehat{\beta}_{F 0}-\widehat{\beta}_{f 0}}{\widehat{\beta}_{f 1}} \leq T_{f}-\frac{\widehat{\beta}_{F 0}-\widehat{\beta}_{f 0}}{\widehat{\beta}_{f 1}}=T_{f^{\star}}\right\}, & \widehat{\beta}_{f 0} \leq \widehat{\beta}_{F 0}\end{cases}
$$

( $F$ analogue). For the comparison of range and spectrum the forces are additionally detrended, so that

$$
\tilde{f}=\left\{f_{t_{i}}^{\star}-\widehat{\beta}_{f^{\star} 0}-\widehat{\beta}_{f^{\star} 1} t_{i} \mid 0 \leq t_{i} \leq \min \left\{T_{f^{\star}}, T_{F^{\star}}\right\}=T_{\tilde{f}}\right\}
$$

with $f^{\star}=\beta_{f^{\star} 0}+\beta_{f^{\star} 1} t+\varepsilon_{f^{\star}}(F$ analogue $)$. Then the range difference is

$$
d_{R}(\tilde{f}, \widetilde{F})=\left|\max _{0 \leq t \leq T_{\tilde{f}}} \widetilde{f}_{t}-\min _{0 \leq t \leq T_{\tilde{f}}} \widetilde{f}_{t}-\max _{0 \leq t \leq T_{\tilde{F}}} \widetilde{F}_{t}+\min _{0 \leq t \leq T_{\tilde{F}}} \widetilde{F}_{t}\right|
$$

and the slope difference is

$$
d_{\beta}\left(f^{\star}, F^{\star}\right)=\left|\widehat{\beta}_{f^{\star} 1}-\widehat{\beta}_{F^{\star} 1}\right| .
$$

Since the modelled sampling rate

$$
\nu_{F}=\frac{\nu v_{c} 10^{3}}{2 \pi r 60} \quad\left[\frac{1}{s}\right]
$$

is much smaller than the sampling rate $\nu_{f}\left[\frac{1}{s}\right]$ and we consider both time series on the same time interval, the number of considered observations $n_{\widetilde{F}}$ of $F$ is also smaller than $n_{\tilde{f}}$. Therefore, the spectral differences are only calculated at the Fourier frequencies

$$
\varphi_{j}=\frac{j}{n} \text { with } n=n_{\widetilde{F}}+\left|\min _{(a, b, c) \in \mathbb{N}^{3}} n_{\widetilde{F}}-2^{a} 3^{b} 5^{c}\right| \text { and } j=1, \ldots,\left\lfloor\frac{n}{2}\right\rfloor
$$

of the shorter time series $\widetilde{F}$. This approach allows the application of the fast Fourier transform (FFT, [2]) algorithm, which by itself enables a fast computation of the periodogram

$$
I_{\widetilde{F}}\left(\varphi_{j}\right)=\frac{1}{\nu_{F} n_{\widetilde{F}}}\left|\sum_{k=1}^{n_{\widetilde{F}}} \widetilde{F}_{k} \exp \left(-i 2 \pi \varphi_{j} k\right)\right|^{2}
$$

as an estimate of the spectrum of $\widetilde{F}$. By adjusting the angular frequencies $2 \pi \varphi_{j}$ to the sampling rate of the measured signal $\widetilde{f}$, we obtain the periodogram

$$
I_{\widetilde{f}}\left(\varphi_{j}\right)=\frac{1}{\nu_{f} n_{\widetilde{f}}}\left|\sum_{k=1}^{n_{\widetilde{f}}} \widetilde{f}_{k} \exp \left(-i 2 \pi \varphi_{j} \frac{\nu_{F}}{\nu_{f}} k\right)\right|^{2}
$$


of $\tilde{f}$ at the same frequencies $\varphi_{j}$ and can determine the spectral differences

$$
d_{S}(\widetilde{f}, \widetilde{F})=\sum_{j=1}^{\left\lfloor\frac{n}{2}\right\rfloor}\left|I_{\widetilde{f}}\left(\varphi_{j}\right)-I_{\widetilde{F}}\left(\varphi_{j}\right)\right| .
$$

Due to the high sampling rate of $f$, the calculation of $\bar{D}$ is time consuming. For this reason we make use of model based optimization techniques, where the objective function (here: $\bar{D}$ ) is substituted with a surrogate model that allows a fast evaluation of many parameter constellations $\theta \in \Theta$ [7]. In the sequential optimization the three steps (1) estimation of the surrogate function, (2) prediction of the best parameter setting, (3) evaluation of this setting with the objective function are repeated in each iteration until a stop criterion (e.g. number of iterations) is fulfilled. Before the first estimation is performed, the objective function is evaluated on a space filling design (e.g. Latin hypercube) to provide data for the estimation. In the following iterations this data set is supplemented by the points evaluated in step (3). The procedure used for the model parameter adjustment is summarized in the flowchart below (fig. 4).

Evaluate $\bar{D}$ on initial design $\Theta_{0}$ :

$\bar{D}\left(\theta_{i}\right)=y_{i}$ with $\theta_{i} \in \Theta_{0}, i=1, \ldots, n_{0}$

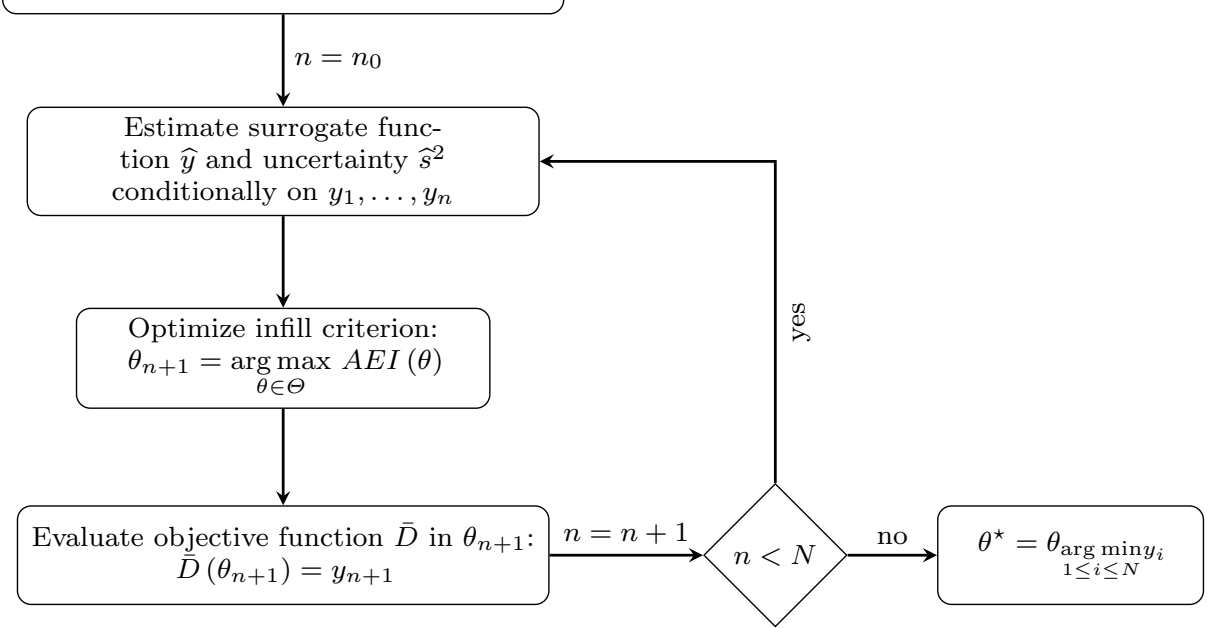

Fig. 4 Steps of the model based optimization used for the adjustment of the model parameters $\theta$ by minimizing the deviation measure $\bar{D}$.

Since the modelled force $\mathcal{F}$ is stochastic, the measure $D$ is also stochastic. In order to take this fact into consideration, we choose the noisy Kriging model as surrogate for $\bar{D}[11]$. Here the process

$$
Y(\theta)=\kappa+H(\theta)+\epsilon, \quad \theta \in \Theta
$$

with constant trend $\kappa$, a Gaussian process $H$ with $E(H(\theta))=0$ and stationary kernel function $h\left(\theta_{i}, \theta_{j}\right)=\delta^{2} \rho\left(\theta_{i}-\theta_{j}, \varphi\right)$ and $\epsilon \sim N\left(0, \tau^{2}\right)$ represents the underlying 
process of $\bar{D}$. Let denote $k\left(\theta_{i}, \theta_{j}\right)=\delta^{2} \rho\left(\theta_{i}-\theta_{j}, \varphi\right)+\tau^{2} \mathbb{1}_{\theta_{i}=\theta_{j}}$ the covariance function of the process $Y$. For $n$ design points $\theta_{1}, \ldots, \theta_{n}$ the variables $Y\left(\theta_{1}\right), \ldots, Y\left(\theta_{n}\right)$ follow the joint distribution

$$
\begin{aligned}
Y & =\left(\begin{array}{c}
Y\left(\theta_{1}\right) \\
\vdots \\
Y\left(\theta_{n}\right)
\end{array}\right)=1 \kappa+\left(\begin{array}{c}
H\left(\theta_{1}\right) \\
\vdots \\
H\left(\theta_{n}\right)
\end{array}\right)+\left(\begin{array}{c}
\epsilon_{1} \\
\vdots \\
\epsilon_{n}
\end{array}\right)=1 \kappa+H+\varepsilon \\
& \sim N\left(1 \kappa, \delta^{2} R(\varphi)+\tau^{2} I_{n \times n}\right)=N(1 \kappa, K)
\end{aligned}
$$

with covariance matrix

$$
K=\left(\begin{array}{c}
\boldsymbol{k}\left(\theta_{1}\right)^{T} \\
\vdots \\
\boldsymbol{k}\left(\theta_{n}\right)^{T}
\end{array}\right)
$$

consisting of the covariance vectors $\boldsymbol{k}\left(\theta_{i}\right)=\left[k\left(\theta_{i}, \theta_{1}\right), \ldots, k\left(\theta_{i}, \theta_{n}\right)\right]^{T}$ between the observations corresponding to the points $\theta_{i}$ and $\theta_{1}, \ldots, \theta_{n}$. Minimizing the mean squared prediction error under the condition $E(Y(\theta))=E(\widehat{Y}(\theta))$ provides the Kriging predictor

$$
\widehat{Y}(\theta)=E(Y(\theta) \mid Y)=\widehat{\kappa}+\boldsymbol{k}(\theta)^{T} K^{-1}[Y-1 \widehat{\kappa}]
$$

with the estimate $\widehat{\kappa}=\frac{1^{T} K^{-1} Y}{1^{T} K^{-1} 1}$ for $\kappa$ and the Kriging variance

$$
\begin{aligned}
\widehat{s}^{2}(\theta) & =\operatorname{Var}(Y(\theta) \mid Y) \\
& =k(x, x)-\boldsymbol{k}(\theta)^{T} K^{-1} \boldsymbol{k}(\theta)+\frac{\left(1-1^{T} K^{-1} \boldsymbol{k}(\theta)\right)^{2}}{1^{T} K^{-1} 1}
\end{aligned}
$$

for each parameter constellation $\theta \in \Theta$. The parameters $\delta^{2}, \tau^{2}, \varphi$ can be estimated by maximizing the likelihood of $Y$. Let denote $\widehat{y}(\theta)=\widehat{E}(Y(\theta) \mid Y=y)$ the Kriging prediction and $\widehat{s}_{y}^{2}(\theta)=\widehat{\operatorname{Var}}(Y(\theta) \mid Y=y)$ the variance estimated on the values $y=\left(y_{1}, \ldots, y_{n}\right)^{T}$ of the objective function for the points $\theta_{1}, \ldots, \theta_{n}$. The next point $\theta_{n+1}$ for the evaluation with the objective function is chosen by maximizing the augmented expected improvement [6]

$$
A E I(\theta)=E I_{\theta_{\min }}(\theta)\left[1-\widehat{\tau}_{y}\left(\widehat{s}_{y}^{2}(\theta)+\widehat{\tau}_{y}^{2}\right)^{-\frac{1}{2}}\right]
$$

over $\theta \in \Theta$ where

$$
\begin{aligned}
E I_{\theta_{\min }}(\theta)= & E\left(\max \left\{\left(\widehat{y}\left(\theta_{\min }\right)-Y(\theta) \mid Y=y, 0\right\}\right)\right. \\
= & \left(\widehat{y}\left(\theta_{\min }\right)-\widehat{y}(\theta)\right) \Phi\left(\frac{\widehat{y}\left(\theta_{\min }\right)-\widehat{y}(\theta)}{\widehat{s}_{y}(\theta)}\right) \\
& +\widehat{s}_{y}(\theta) \phi\left(\frac{\widehat{y}\left(\theta_{\min }\right)-\widehat{y}(\theta)}{\widehat{s}_{y}(\theta)}\right)
\end{aligned}
$$

is the expected improvement over the effective best solution

$$
\theta_{\min }=\underset{\theta \in\left\{\theta_{1}, \ldots, \theta_{n}\right\}}{\arg \min } \widehat{y}(\theta)+\eta \widehat{s}_{y}(\theta)
$$


with $\Phi$ the Gaussian cumulative distribution function, $\phi$ the Gaussian density function, a parameter $\eta$ (here: 1 ) and $\widehat{\tau}_{y}^{2}$ the estimated value for the error variance $\tau^{2}$. The optimization of this infill criterion $A E I$ is solved by the focus search procedure [1]. In this procedure the parameter space $\Theta$ is gradually limited around the points with high values of the augmented expected improvement. For the exploration of the parameter space and each considered subspace points are sampled from a random Latin hypercube design [8]. The next point is chosen as the point of any subspace with the maximal observed infill criterion.

The described optimization is performed for each of the sixteen speed combinations of the full factorial design (see sec. 2) with the R package "mlrMBO" ([12], [1]). Each optimization starts with a Latin hypercube design consisting out of $n_{0}=80$ points $\theta_{1}, \ldots, \theta_{80}$. During the optimization 720 further points are found using the augmented expected improvement and evaluated with the objective function. As covariance function $h$ the anisotropic Matérn(5/2) kernel with different range parameters $\varphi_{1}, \ldots, \varphi_{8}$ for the eight dimensions is chosen.

\section{Results}

Figure 5 shows 50 realizations of the force model with the optimal parameter setting found with the model based optimization methods described in the previous section. The fit between the observed force (black) and modelled forces (blue, transparent) is very promising. The goodness of each fit is unfortunately related to the degree of congruence of the observed forces for the repetitions of the radii $r \in \mathcal{R}\left(v_{c}, v_{f}\right)$ (see sec. 2). If the discrepancies between the repetitions are too strong, the radius parameter in the model is not able to smooth out the resulting variation and the fit becomes poor. Since in the most cases this issue is not relevant, we use the optimized model for the analysis of the influence between the speed parameters and the conducted physical work.

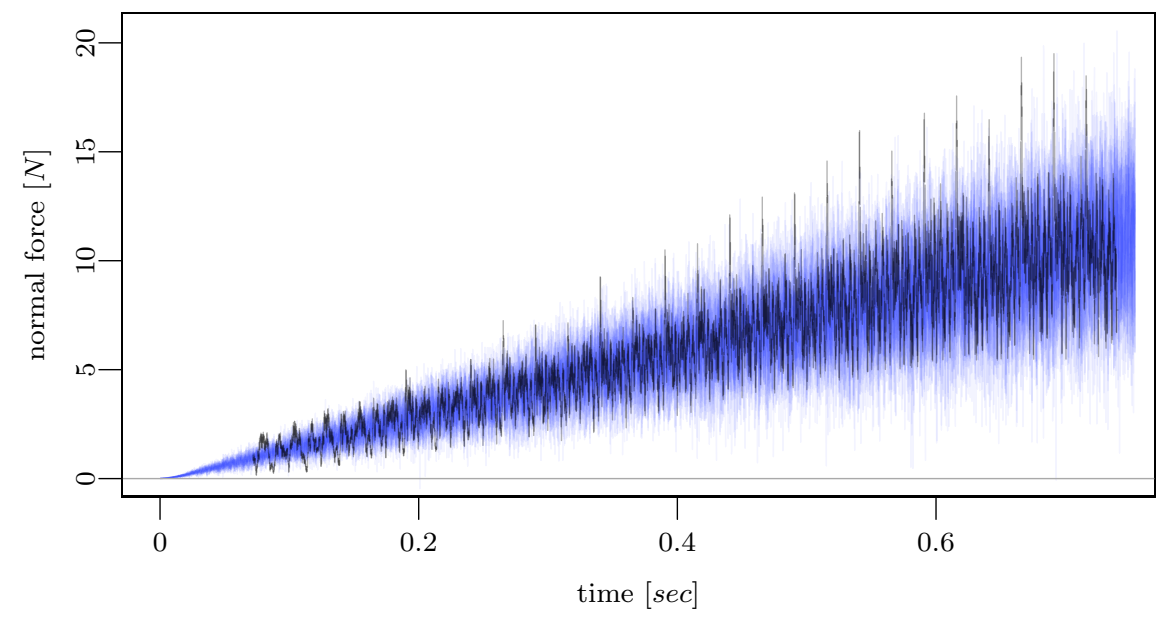

Fig. 5 Fifty realizations of the force model with optimal parameter setting (blue, transparent) and observed force (black) for the speed combination $v_{c}=270 \frac{\mathrm{m}}{\mathrm{min}}$ and $v_{f}=7 \frac{\mathrm{mm}}{\mathrm{min}}$ on the radius of $18 \mathrm{~mm}$. 
For this analysis we use the force model to predict the best speed combination with minimal work needed to drill a desired depth $A$ (here: $A=0.07 \mathrm{~mm}$ ). Therefore, the work

$$
W\left(v_{c}, v_{f}, r\right)=\int_{0}^{s(A)} \bar{F}_{s}\left(\theta^{\star}\left(v_{c}, v_{f}\right), r\right) d s
$$

with the covered distance

$$
s(A)=10^{-3} \frac{A}{a} \sqrt{\left(\frac{2 \pi r}{\nu}\right)^{2}+a^{2}}
$$

in meters for the drilling depth of $A m m$ is approximated. The force $\bar{F}_{s}$ for the covered distance $s=s(t)=\left(t \frac{1000 v_{c} \nu}{602 \pi r}-\frac{1}{2}\right) \sqrt{\left(\frac{2 \pi r}{\nu}\right)^{2}+a^{2}}\left(s\left(t_{0}\right)=0\right)$ is calculated in the same way as $F$, but with the expected triangle height $E\left(h_{j}\right)=(j-2) a+$ $E\left(a^{\star}\right)(j>1)$ in equation $(2)$ and $(5)$ and the expected heterogeneity $z_{i}=\mu$ in equation (9). In figure 6 (a) the force $\bar{F}_{s}$ (red curve) is shown exemplarily for one of the parameter settings $\left(v_{c}, v_{f}, r\right)$. The red hatched area under the curve of $\bar{F}_{s}$ is the work corresponding to the drilling depth of $0.07 \mathrm{~mm}$. After determining the work for all different parameter settings $\left(v_{c}, v_{f}, r\right)$ described in section 2 , a generalized linear model with the log-link and gamma distributed errors is fitted to the data considering linear, quadratic and cubic effects as well as all interactions of $v_{c}, v_{f}$ and $r$. The predicted surface of the selected model

$$
\begin{aligned}
W= & \beta_{1} v_{c}+\beta_{2} v_{f}+\beta_{3} r+\beta_{4} v_{c}^{2}+\beta_{5} v_{f}^{2}+\beta_{6} r^{2}+\beta_{7} v_{c}^{3}+\beta_{8} v_{f}^{3}+\beta_{9} r^{3} \\
& +\beta_{10} v_{c}^{2} v_{f}+\beta_{11} v_{f}^{2} v_{c}+\beta_{12} v_{f}^{2} r+\beta_{13} r^{2} v_{c}+\beta_{14} r^{2} v_{f} \\
& +\beta_{15} v_{c}^{2} v_{f}^{2}+\beta_{16} r^{2} v_{f}^{2}+\beta_{17} r^{2} v_{c}^{2} \\
& +\beta_{18} v_{c}^{3} v_{f}+\beta_{19} v_{c}^{3} r+\beta_{20} v_{f}^{3} v_{c}+\beta_{21} r^{3} v_{c}+\beta_{22} r^{3} v_{f}+\varepsilon_{W}
\end{aligned}
$$

(AIC, backwards, $R_{\text {pseudo }}^{2}=0.994$ ) is shown in figure $6(\mathrm{~b})$.

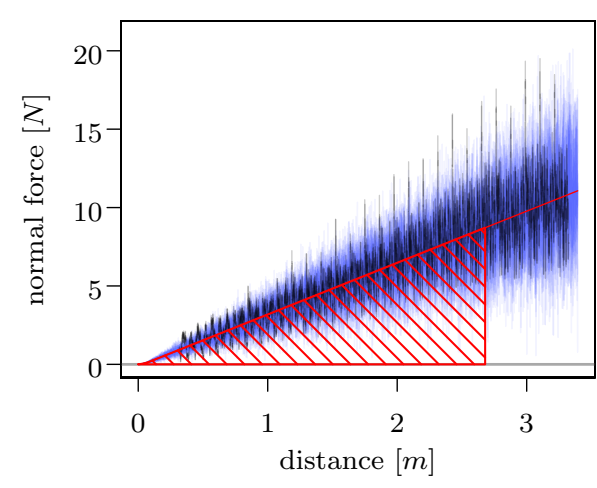

(a)

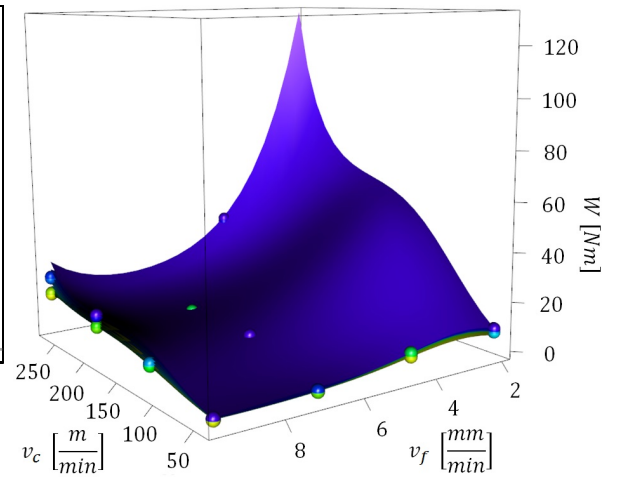

(b)

Fig. 6 (a) Realizations of the force model (blue), observed force (black) and the resulting work (red hatched) to reach a total depth of $A=0.07 \mathrm{~mm}$ with $\left(v_{c}, v_{f}, r\right)=$ $\left(270 \frac{\mathrm{m}}{\mathrm{min}}, 7 \frac{\mathrm{mm}}{\mathrm{min}}, 18 \mathrm{~mm}\right)$ and (b) Surface of the generalized linear model of the resulting work split up by the radii $r=16 \mathrm{~mm}$ (yellow), ..., $r=27 \mathrm{~mm}$ (violet). 
The surface is plotted separately for the twelve radii. The minimal work needed to drill $A=0.07 \mathrm{~mm}$ is identified for all radii at a cutting speed of $v_{c}=40.5 \frac{\mathrm{m}}{\mathrm{min}}$ and an average feed speed $v_{f}=8.734 \frac{\mathrm{mm}}{\mathrm{min}}(s d=0.074)$. This outcome suggests that choosing a small cutting speed in combination with higher feed speed results in benefits concerning the work. Since $v_{f}=8.734$ is not the maximal value of $v_{f}$ considered in the design of experiments, it is assumable that values of $v_{f}$ higher than the found optimum will cause more work. This result is plausible since too small feed speeds lead to a long process time which results in higher work and too high feed speeds lead to a high material removal rate which in turn results in higher work as well.

\section{Conclusion and future Work}

In the presented work we derived a model for the simulation of forces arising during a diamond drilling process. With the model based optimization techniques for stochastic black box functions, a realistic outcome could be achieved for the model with adjusted model parameters. Finally, the optimized force model was used for the process optimization. It was determined that lower cutting speed results in lower work needed to drill a predefined depth, while the feed speed has to be chosen in average at $v_{f}=8.734 \frac{\mathrm{mm}}{\mathrm{min}}$. This result is nearly the same for all examined drilling radii, which is a satisfactory outcome since in the most situations the drilling radius is determined by the type of application and is not freely selectable.

Due to the possibility of a fast computation of forces in a single diamond drilling process, we will extend the model for the multiple diamond drilling. This process is, as well as the diamond grinding, an often considered sub-process of the core drilling, where multiple diamonds and metal powder form a segment. Segment drilling introduces further parameters for the description of the interaction between diamond and workpiece. Not all diamonds have contact to the workpiece from the beginning of the process and not all diamonds are still remaining in the segment when the process is terminated. The diamond-workpiece-contact-time is as important as the sizes and angles of the diamonds. Force time series of already conducted segment experiments show a high increase of forces when multiple diamonds come into contact with the workpiece surface. Thus, it seems obvious that the forces in a segment experiment can be explained as the sum of forces of the single diamonds in this segment. The occurrence of stationary periods suggests that the contact area between diamonds and workpiece is constant after reaching a maximum which depends on the sizes and angles of the diamonds. The greatest challenge in deriving the segment drilling model is thus to find an appropriate model for the on- and offset of the diamond-workpiece contact.

Additionally, we are working on the extension of the model for composite materials like concrete. The presented optimized model is adjusted for data from experiments on cement. Since concrete is a composite material consisting out of cement and an aggregate, e.g. basalt, the model parameter adjustment results in a multi-objective optimization problem, because the parameters $g_{z v}, g_{v}$ have to fit for both materials, while the others can be material specific. 
Acknowledgements This work has been supported by the Collaborative Research Center "Statistical modeling of nonlinear dynamic processes" (SFB 823) of the German Research Foundation (DFG).

\section{References}

1. Bischl, B., Bossek, J., Horn, D., Lang, M.: mlrMBO: Model-based Optimization for mlr. URL https://github.com/berndbischl/mlrMBO. R package version 1.0

2. Bloomfield, P.: Fourier Analysis of Time Series: An Introduction. John Wiley \& Sons (2004)

3. Brinksmeier, E., Aurich, J.C., Govekar, C., Hoffmeister, H.W., Klocke, F., Peters, J., Rentsch, R., Stephenson, D.J., Uhlmann, E., Weinert, K., Wittmann, M.: Advances in modeling and simulation of grinding processes. CIRP Annals-Manufacturing Technology $\mathbf{5 5}(2), 667-696(2006)$

4. Franca, L.F.P., Mostofi, M., Richard, T.: Interface laws for impregnated diamond tools for a given state of wear. International Journal of Rock Mechanics and Mining Sciences 73, 184-193 (2015)

5. Herbrandt, S., Weihs, C., Ligges, U., Ferreira, M., Rautert, C., Biermann, D., Tillmann, W.: Optimization of a simulation for inhomogeneous mineral subsoil machining. In: A. Wilhelm, H. Kestler (eds.) Analysis of Large and Complex Data. Springer (2016)

6. Huang, D., Allen, T.T., Notz, W.I., Zeng, N.: Global optimization of stochastic blackbox systems via sequential Kriging meta-models. Journal of global Optimization 34(3), 441-466 (2006)

7. Jones, D.R., Schonlau, M., Welch, W.J.: Efficient global optimization of expensive blackbox functions. Journal of global Optimization 13(4), 455-492 (1998)

8. Li, Z., Nakayama, S.: Maximin distance-lattice hypercube design for computer experiment based on genetic algorithm. In: Y.X. Zhong, J. Shi, X. Lin (eds.) 2001 International Conferences on Info-tech and Info-net Proceedings. ICII 2001-Beijing, China, vol. 2, pp. 814-819. IEEE (2001)

9. M, S., A, M., M, O., D, B., K, S., S, E., J, M., F, B., O, M., PJ, M., S, G., U, O., Berreth, C., K, B., J, M., O, M., P, R., R, S., B, P., Team, R.C.: RandomFields. URL http://CRAN.R-project.org/package=RandomFields. R package version 3.1.4

10. Maur, P.: Delaunay triangulation in 3d. Tech. rep., Technical Report No. DCSE/TR-200202, University of West Bohemia in Pilsen (2002)

11. Picheny, V., Wagner, T., Ginsbourger, D.: A benchmark of Kriging-based infill criteria for noisy optimization. Structural and Multidisciplinary Optimization 48(3), 607-626 (2013)

12. R Core Team: R: A Language and Environment for Statistical Computing. R Foundation for Statistical Computing, Vienna, Austria (2015). URL http://www.R-project.org/

13. Raabe, N., Rautert, C., Ferreira, M., Weihs, C.: Geometrical process modeling and simulation of concrete machining based on Delaunay tessellations. In: S.I. Ao, C. Douglas, W.S. Grundfest, J. Burgstone (eds.) Proceedings of the World Congress on Engineering and Computer Science 2011 Vol II, WCECS '11, October 19 - 21, 2011, San Francisco, USA, Lecture Notes in Engineering and Computer Science, pp. 991-996. International Association of Engineers, Newswood Limited (2011)

14. Raabe, N., Thieler, A.M., Weihs, C., Fried, R., Rautert, C., Biermann, D.: Modeling material heterogeneity by Gaussian random fields for the simulation of inhomogeneous mineral subsoil machining. In: P. Dini, P. Lorenz (eds.) SIMUL 2012: The Fourth International Conference on Advances in System Simulation, November 18 - 23, 2012, Lisbon, Portugal, pp. $97-102(2012)$

15. Weihs, C., Raabe, N., Ferreira, M., Rautert, C.: Statistical process modelling for machining of inhomogeneous mineral subsoil. In: W. Gaul, A. Geyer-Schulz, Y. Baba, A. Okada (eds.) German-Japanese Interchange of Data Analysis Results, pp. 253-263. Springer (2014)

16. Wheeler, R.E.: Comments on algorithmic design. Vignette accompanying package AlgDe$\operatorname{sign}(2004)$ 


